\title{
Carbonates in Planetary Nebulae
}

\author{
F. Kemper, L. B. F. M. Waters, A. de Koter
}

Astronomical Institute 'Anton Pannekoek', University of Amsterdam, Kruislaan 403, 1098 SJ Amsterdam, The Netherlands

C. Jäger, Th. Henning

AIU, University of Jena, Germany

F. J. Molster

ESTEC/ESA, Solar system division, Noordwijk, The Netherlands

M. J. Barlow

Dept. of Physics and Astronomy, University College London, UK

T. Lim

Space Science Department, Rutherford Appleton Laboratory, UK

We report the discovery of carbonates in the Planetary Nebulae NGC 6302 and NGC 6537 (Kemper et al. 2002). In the ISO LWS spectra far-infrared features have been identified with calcite and dolomite by comparison with laboratory spectra of these minerals. This is the first time that carbonates have been identified outside the solar system. In a follow-up study (Kemper et al., in prep.) a detailed analysis of the mineral composition of the dust in NGC 6302 is presented.

Carbonates are very common on earth and also frequently found in the solar system, for example in interplanetary dust particles (IDPs). It is generally thought that the formation of carbonates proceeds through weathering of silicates with a watery $\left(\mathrm{CO}_{3}\right)^{2-}$ solution, a process called aqueous alteration. Consequently, IDPs containing carbonates are thought to be processed on a large parent body located in the inner regions of the solar system. The lack of large parent bodies and liquid water in planetary nebulae implies that an alternative formation mechanism exists.

\section{References}

Kemper, F., Jäger, C., Waters, L. B. F. M., Henning, Th., Molster, F. J., Barlow, M. J., Lim, T. \& de Koter, A. 2002, Nature, 415, 295 